\title{
Article
}

\section{Ethnomedicinal survey of plants used by local society in Poncokusumo district, Malang, East Java Province, Indonesia}

\author{
Jati Batoro*and Dian Siswanto \\ Biology Departement Faculty of Mathematics and Natural Sciences Brawijaya University Adress: Jl. Veteran \\ Malang 65145 East Java, Indonesia \\ *Corresponding author: Jati Batoro, Biology Department, Faculty of Mathematics and Natural Sciences, \\ Brawijaya University, Jl. Veteran Malang 65145 East Java, Indonesia. Telp-fax: +62-341-575841; E-mail: \\ jati_batoro@yahoo.co.id
}

Received: 16 May 2017/Accepted: 31 May 2017/ Published: 29 June 2017

\begin{abstract}
An ethnomedicinal survey was carried out in district Poncokusumo, Malang, East Java Province, Indonesia for documentation of important plants diversity and information from local society. The indigenous knowledge of traditional local societywas collected throught structural and open indept interview, direct observation and personal interviews during the research. To better acessesto the extractive activities and the utilization of the plant diversity by indigenous people. Plants with their correct nomenclature were arranged by vernacular name, family name, parts used, ethnomedicinal remedies and ethnomedical use. The determination and nomenclature of the listed plants were based on the Flora of Java.A Total of 181 species plants (68 family) determinate of Tengger and Java people existing in the region. Family ethnomedicine plants that have large members includes Umbelliferae (3 species), Apocynaceae (4 species), Gramineae (6 species), Myrtaceae (7 species), Euphorbiaceae (8 species), Fabaceae (10 species), Zingiberaceae (10 species), Solanaceae (12 species) and Asteraceae ( 15 species).The number of plants used to treat more than 60 diseases. The treatment done by a medicine man or shaman from Tengger people by ritual treatment with called "Suwuk" .
\end{abstract}

Keywords: ethnomedicinal plants; indigenous knowledge; local people; district Poncokusumo Malang; East Java

\section{Introduction}

Indonesia is an important country which has "mega-biodiversity" and its variety of culture becoming unique, attractive and having high potention resources that were not yet explored, known and exploited. These are all used for supporting people's life. The wisdom utilization of them can be functioned as the people welfare in the future. More thanthree thousand species and variety of flowering plants are reported from Java land Indonesia (Backer and van den Brink, 1968). Rifai (1994) reported at least 940 species of plants are currently being used in traditional medicines. Many of these species are useful as tonics and prophylactics to help keep the body fit. Besides the familiar microbial, fungi, algae resource, Volvariella volvacea, Usnea barbata, etc. Medicinal plants play a key role in traditional health care system for human and animals and most of allopathic drugs also comprise extracts taken from medicinal plants. According to Claudine Berthe-Frieberg in Waluyo (2004) describes as two basic approaches that must be considered in the study ethnomedicine follow perception and conception ofan object approaches and scientific fields.

Ethnobotany methods done with exploratory surveys namely biodiversity in ventory drugs in the community. Research can be integrated with technique for example Rapid Rural Appraisal (PRA), Particypatory Rural Appraisal and Rapid Ethnobotanical Appraisal (REA) (Cotton, 1996; Hoffman and Gallaher, 2007). Ethnomedicines acts as a bridge between botany and tribal knowledge regarding medicinal aspects of plants. 
Tengger people added good knowledge and important ancient source of information on medicinal plants. The modern literature has further added to our knowledge regarding plant-based remedies.

The total area of the district Poncokusumo is 209.888 hectare, and has 4 villages. Tengger people in district Poncokusumo involved five villages viz Poncokusumo, Pandansari, Duwet, Gubuklakah and Ngadas and Java people has 2 villages namely Wringinanom and Sumberejo (Stibbe and Uhlenbeck, 1921; Batoro, 2015). In the east by the Senduro district, in the south by the Wajak district, in the north by the Tajinan district, and in the west by the Tumpang district (Fig. 1A). The district Poncokusumotemperature between $10^{\circ} \mathrm{C}-22^{\circ} \mathrm{Cand}$ has latitudes between $700 \mathrm{~m} \mathrm{dpl}-1800 \mathrm{~m}$ dpl. In the Ngadas village (inclave) is Tengger people bounded Bromo Tengger Semeru National Park (BTSNP) (Fig. 1B).To protect the importance of medicinal flora Tengger people in district Poncokusumo Malang, Province East Java Indonesia conservation must be realize. This study was arranged to document and collect ethnomedicinaltibb and ethnomedicinal knowledge about the wild plants and agricultural of Poncokusumo distric-Malang city.

\section{Materials and Methods}

\subsection{Preservation and sample collection}

The research were arranged in order to collect information about the Ethnomedicinal tibb and ethnomedicinal uses of plants by the Tengger and Java people during 2013-2015 in district Poncokusumo Malang, East Java, Indonesia. Standard methods were followed with regard for collection of plant materials, drying, mounting, preparation and preservation of plant specimens.Herbarium specimens of medicinal plants in triplicates were collected, prepared and determinated. Plant spesimens collected identificated, preseved and mounted were deposited in Herbarium of Brawijaya University (H. Bio Unibraw) Malang, East Java, Indonesia.This study concerning about local people's knowledge about medicine (Ethnomedicine) in Poncokusumo district, Malang, East Java, Indonesia.

Plants with their correct nomenclature were arranged alphabetically by local name, scientific name, family name, ethnomedicinaltibb and ethnomedicinal uses. The determination and nomenclature of the listed plants were based on the Flora of Java (Backer and van den Brink, 1968; Chinery, 1982; De Vogel, 1987).

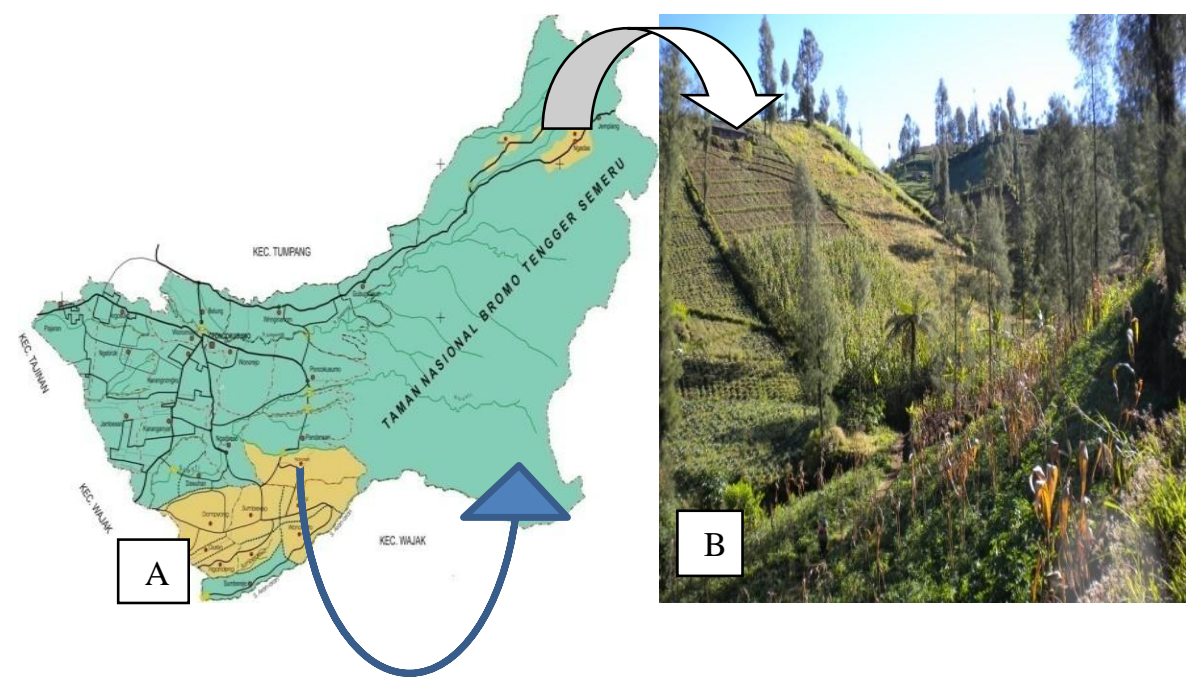

Figure 1.A. Map of district Poncokusumo Malang, East Java and locations of study sites (TAMAN NASIONAL BROMO TENGGER SEMERU is mentioned as Bromo Tengger National Park (BTS NP) in this paper). B. Ngadas village is an inclave Village in Bromo Tengger National Park (BTS NP).

\subsection{Ethnomedicinal knowledge}

A questionnaire method was adopted for documentation of ethnomedicinal knowledge Tengger society. The interviews were carried out from local community to document botany local name and ethnomedicinal uses. About 42 informants have been interviewed on random base (Cotton, 1996; Sheil et al., 2004; Waluyo, 2004). The indigenous medicinal plants having traditional knowledge (perception and conception) of utilization among the Tengger and Java societyhave been selected as reference speciments herbarium. 
3. Results and Discussion

During the present study, ethnomedicinal data on 181 plant species was collected and preserved at Herbarium of Brawijaya University (H Bio Unibraw) (Table 1). From the total colected ethnomedical plants belonging to150 genera and 66 families which were recorded. Information regarding their vernacular name, botanical name, family, part used and their ethnomedicinal uses are listed below starting with local name, scientific name and family name, part used and Ethomedicinal uses.

Table 1. Presentage of life form of ethnomedicinal plant use by local people district Poncokusumo Malang.

\begin{tabular}{|c|c|c|c|c|c|}
\hline No & Local name & Scientific name & Family & Parts used & Ethnomedicinal uses \\
\hline 1 & Aseman & $\begin{array}{l}\text { Achiranthes } \\
\text { bidentata } \mathrm{B} 1 .\end{array}$ & $\begin{array}{l}\text { Amaranthace } \\
\text { ae }\end{array}$ & Young stem, leaf & $\begin{array}{l}\text { Kidney problems and } \\
\text { cough,inflamations, } \\
\text { gonorrhea,headache }\end{array}$ \\
\hline 2 & Bayam & $\begin{array}{l}\text { Amaranthus hybridus } \\
\text { L. }\end{array}$ & $\begin{array}{l}\text { Amaranthace } \\
\text { ae }\end{array}$ & Young stem, leaf & $\begin{array}{l}\text { Vitality,inflamations, piles, } \\
\text { gonorrhea,hipertension and skin } \\
\text { allergies }\end{array}$ \\
\hline 3 & Mangga & $\begin{array}{l}\text { Mangifera indica } \mathrm{L} \text {. } \\
\text { cv. Manalagi }\end{array}$ & $\begin{array}{l}\text { Anacardiacea } \\
\mathrm{e}\end{array}$ & Fruit & $\begin{array}{l}\text { Stomach acidity and skin } \\
\text { allergy, hemorrhoid }\end{array}$ \\
\hline 4 & Mangga & $\begin{array}{l}\text { Mangifera indica L. } \\
\text { cv. Gadung }\end{array}$ & $\begin{array}{l}\text { Anacardiacea } \\
\text { e }\end{array}$ & Leaf, friut & Ear ache, hemorrhoid, vomiting \\
\hline 5 & Sirsat & Annona muricata $\mathrm{L}$. & Annonaceae & Leaf, fruit & $\begin{array}{l}\text { Reumatik, hypertension, skin } \\
\text { diseases \&helminthiasis }\end{array}$ \\
\hline 6 & Srikoyo & Annona squamosa $\mathrm{L}$. & Annonaceae & Fruit & Reumatik, hemorrhoid \\
\hline 7 & Kenongo & $\begin{array}{l}\text { Cananga odorata } \\
\text { Hook.f.\& Th. }\end{array}$ & Annonaceae & Flower, leaf & Ritual, obsession, hair oil \\
\hline 8 & Sledri & Apium graviolens L. & Apiaceae & $\begin{array}{l}\text { Yung stem, leaf, } \\
\text { fruit }\end{array}$ & $\begin{array}{l}\text { Hypertension, the smell of } \\
\text { sweat, foodflavoring, headache }\end{array}$ \\
\hline 9 & Calingan & Centela asiatica Urb. & Apiaceae & Whole plant & Cough, urinary,stones \\
\hline 10 & Tumbar & $\begin{array}{l}\text { Coriandrum sativum } \\
\text { L. }\end{array}$ & Apiaceae & Fruit & Cold, stimulan \\
\hline 11 & Wortel & Daucus carota $\mathrm{L}$. & Apiaceae & Whole plant & Sprue, eye treatment \\
\hline 12 & Pule & $\begin{array}{l}\text { Alstonia shcolaris } \\
\text { R.Br. }\end{array}$ & Apocynaceae & Stem & Injury, headaches \\
\hline 13 & Pulosari & Alyxia reinwardii $\mathrm{L}$. & Apocynaceae & Leaf, fruit, stem & In the treatment of asthma. \\
\hline 14 & Ampet & $\begin{array}{l}\text { Astronia macrophilla } \\
\text { L. }\end{array}$ & Apocynaceae & Stem & Dysentery \\
\hline 15 & Tapak doro & $\begin{array}{l}\text { Catharanthus roseus } \\
\text { (L.) G.Don. }\end{array}$ & Apocynaceae & $\begin{array}{l}\text { Young stem, leaf, } \\
\text { flower }\end{array}$ & Diabetes mellitus \\
\hline 16 & Dringu & Acorus calamus $\mathrm{L}$. & Araceae & Leaf, rhizome & $\begin{array}{l}\text { Asthma, cough tuberculosis, } \\
\text { bloated }\end{array}$ \\
\hline 17 & Mbote & $\begin{array}{l}\text { Calocasia esculentum } \\
\text { Schott. }\end{array}$ & Araceae & Stem & $\begin{array}{l}\text { It is effective against cancer } \\
\text { and cure mouth and feet } \\
\text { diseases, sleep }\end{array}$ \\
\hline 18 & Bentul & $\begin{array}{l}\text { Xanthosoma } \\
\text { violacium } \text { Schott }\end{array}$ & Araceae & Stem & Vitality \\
\hline 19 & Sangit & Eryngium foetidum $\mathrm{L}$. & Araliaceae & Whole plant & Diabetes mellitus \\
\hline 20 & Cakar kucing & $\begin{array}{l}\text { Polyscias fructicosa } \\
\text { (L.) Harms. }\end{array}$ & Araliaceae & leaf & Vitality \\
\hline 21 & Aren & $\begin{array}{l}\text { Arenga pinnata } \\
\text { (Wurm.) Merr. }\end{array}$ & Arecaceae & fruit & Hypertension and skin allergies \\
\hline 22 & Rotan & Calamus javensis Bl. & Arecaceae & $\begin{array}{l}\text { Young stem } \\
\text { (umbut) }\end{array}$ & Dysentery \\
\hline 23 & Klopo & Cocos nucifera $\mathrm{L}$. & Arecaceae & Fruit, water & Soap, margarine \\
\hline 24 & Piji & $\begin{array}{l}\text { Pinanga coronata } \\
\text { (B1.ex Mart.) Bl. }\end{array}$ & Arecaceae & $\begin{array}{l}\text { Young stem } \\
\text { (umbut) }\end{array}$ & Dysentery, ritual Tengger \\
\hline 25 & Salak & Sallaca edulis Reinw & Arecaceae & Fruit & Dysentery \\
\hline 26 & $\begin{array}{l}\text { Jambe/pinan } \\
\mathrm{g}\end{array}$ & Areca catechu L. & Arecaceae & Young stem, fruit & $\begin{array}{l}\text { Protection of teeth, dysentery, } \\
\text { cosmetic, wormy }\end{array}$ \\
\hline 27 & Bandotan & Ageratum sp. & Asteraceae & Whole plant & Toxic \\
\hline
\end{tabular}




\begin{tabular}{|c|c|c|c|c|c|}
\hline 28 & Wedusan & $\begin{array}{l}\text { Ageratum conyzoides } \\
\text { L. }\end{array}$ & Asteraceae & Whole plant & Facilitatingurine, tumor, cancer \\
\hline 29 & Tanalayu & $\begin{array}{l}\text { Anaphalis javanica } \\
\text { (Reinw.) Schulzh.; }\end{array}$ & Asteraceae & Whole plant & Ritual (petra) \\
\hline 30 & Tanalayu & $\begin{array}{l}\text { Anaphalis longifolia } \\
\text { (B1.) DC }\end{array}$ & Asteraceae & Whole plant & Ritual (petra) \\
\hline 31 & Tiu & $\begin{array}{l}\text { Emilia sonchifolia } \\
\text { (L.) DC }\end{array}$ & Asteraceae & Latex leaf,flower & Icth, wound \\
\hline 32 & Kerinyu & $\begin{array}{l}\text { Eupatorium } \\
\text { inulifolium H.B.k }\end{array}$ & Asteraceae & Whole plant & $\begin{array}{l}\text { Toxic/ leaf paste applied to } \\
\text { treat allergy, athlete's foot and } \\
\text { ringworm. }\end{array}$ \\
\hline 33 & Putihan & $\begin{array}{l}\text { Eupatorium } \\
\text { odoratum L.f. }\end{array}$ & Asteraceae & Whole plant & Toxic \\
\hline 34 & Tehan & $\begin{array}{l}\text { Eupatorium riparium } \\
\text { Reg. }\end{array}$ & Asteraceae & Leaf & Toxic \\
\hline 35 & Berokan & $\begin{array}{l}\text { Eupatorium } \\
\text { triplinerve M.Vahl }\end{array}$ & Asteraceae & Whole plant & Toxic \\
\hline 36 & Pusek & $\begin{array}{l}\text { Gynura procumbens } \\
\text { (Lour.) Merr. }\end{array}$ & Asteraceae & Whole plant & Itchdrug \\
\hline 37 & Menjari & $\begin{array}{l}\text { Sonchus javanicus } \\
\text { Jungh. }\end{array}$ & Asteraceae & $\begin{array}{l}\text { Whole plant, } \\
\text { latex }\end{array}$ & Itch, dewormingdrugs \\
\hline 38 & $\begin{array}{l}\text { Nyamu/paita } \\
\text { n/liyer }\end{array}$ & $\begin{array}{l}\text { Tithonia diversifolia } \\
\text { Gray }\end{array}$ & Asteraceae & Whole plant & Toxic, stimulants \\
\hline 39 & Kuningan & $\begin{array}{l}\text { Widelia montana } \\
\text { (B1.) Boerl }\end{array}$ & Asteraceae & Leaf, flowers & $\begin{array}{l}\text { Inflammations, asthma and } \\
\text { diseases. }\end{array}$ \\
\hline 40 & Ganjan & Artemisia vulgaris L. & Asteraceae & Leaf & Toxic \\
\hline 41 & Sempretan & Bidens pilosa $\mathrm{L}$. & Asteraceae & Radix & Asthma, reumatic, vitality \\
\hline 42 & Jamur kuping & $\begin{array}{l}\text { Auricularia } \\
\text { polystrica } \\
\text { (Montagne) } \\
\text { Saccardo. }\end{array}$ & Auriculariaceae & Fruit & Vitality \\
\hline 43 & Binahong & Basella rubra L. & Basellaceae & Young stem, leaf & Wound \\
\hline 44 & Durian & $\begin{array}{l}\text { Durio zibethinus } \\
\text { Murray }\end{array}$ & Bombaceae & Fruit & Hypertension, vitality \\
\hline 45 & Sawi & $\begin{array}{l}\text { Brassica juncea } \\
\text { Cosson }\end{array}$ & Brassicaceae & Leaf & $\begin{array}{l}\text { Wounds, earaches and ulcers. } \\
\text { The seeds are anthelmintic, } \\
\text { carminative, stimulant and } \\
\text { vesicant }\end{array}$ \\
\hline 46 & Kobis & Brassica oleracea L. & Brassicaceae & Leaf & $\begin{array}{l}\text { Wounds, earaches and } \\
\text { ulcers,the seeds are } \\
\text { anthelmintic, carminative, } \\
\text { stimulant and vesicant }\end{array}$ \\
\hline 47 & Lobak & Rhapanus sativus L. & Brassicaceae & Leaf, flower & Gout,flatulence, a bee sting \\
\hline 48 & Nanas & $\begin{array}{l}\text { Ananas comusus } \\
\text { Merr. }\end{array}$ & Bromeliaceae & Young fruit & Used for abortion. \\
\hline 49 & Tepung otot & Plantago mayor L. & Campanulaceae & $\begin{array}{l}\text { Whole plant: } \\
\text { leaf, seed }\end{array}$ & $\begin{array}{l}\text { Sprained, diabetes mellitus, } \\
\text { herbs }\end{array}$ \\
\hline 50 & Kates & Carica papaya $\mathrm{L}$. & Caricaceae & Leaf, fruit & Vitality, hemorrhoid \\
\hline 51 & Srikaya & Carica pubescent $\mathrm{L}$. & Caricaceae & Fruit & Vitality, hemorrhoid \\
\hline 52 & $\begin{array}{l}\text { Cemara } \\
\text { gunung }\end{array}$ & $\begin{array}{l}\text { Casuarina } \\
\text { junghuhniana L. }\end{array}$ & Casuarinaceae & Stem (ash) & Dysentery \\
\hline 53 & Tirem & Solanum sp. & Convolvulaceae & Leaf & Vitality, stimulans, headaches \\
\hline 54 & Timun & Cucumis sativus L. & Cucurbitaceae & Fruit & Hypertention, skin allergies \\
\hline 55 & Waluh & $\begin{array}{l}\text { Cucurbita moschata } \\
\text { (Duch.ex Lam.) } \\
\text { Duch.ex Poir. }\end{array}$ & Cucurbitaceae & Fruit & Vitality \\
\hline 56 & Pare & $\begin{array}{l}\text { Mimordica charantia } \\
\text { L. }\end{array}$ & Cucurbitaceae & Fruit & $\begin{array}{l}\text { Vitality, stimulanmosquito } \\
\text { bites, headaches }\end{array}$ \\
\hline 57 & Siyem & $\begin{array}{l}\text { Sechium edule (Jacq.) } \\
\text { Swart }\end{array}$ & Cucurbitaceae & Fruit & Infections, stomach problems \\
\hline 58 & Teki & Cyperus rotundus $\mathrm{L}$. & Cyperaceae & Rhizomes & $\begin{array}{l}\text { Dysentery and blood disorders, } \\
\text { headaches, tuberous, }\end{array}$ \\
\hline
\end{tabular}




\begin{tabular}{|c|c|c|c|c|c|}
\hline 59 & Kesemek & Diospyros kaki L. & Ebenaceae & Fruit & $\begin{array}{l}\text { stomachic and diuretic } \\
\text { Vitality, hypertension }\end{array}$ \\
\hline 60 & Petungan & $\begin{array}{l}\text { Equisetum debile } \\
\text { Roxb. }\end{array}$ & Equisetaceae & Stem, leaf & Dysentry \\
\hline 61 & Buntut tikus & Acalypha indica $\mathrm{L}$. & Euphorbiaceae & Leaf & Bloated \\
\hline 62 & Kemiri & $\begin{array}{l}\text { Aleruites moluccana } \\
\text { Willd }\end{array}$ & Euphorbiaceae & Seed & Hairgrower, bumbu (seasoning) \\
\hline 63 & $\begin{array}{l}\text { Kontol } \\
\text { belang }\end{array}$ & $\begin{array}{l}\text { Euphorbia } \\
\text { pulcheimaL. }\end{array}$ & Euphorbiaceae & $\begin{array}{l}\text { Whole plant, } \\
\text { latex }\end{array}$ & $\begin{array}{l}\text { Expectroant, used in bronchitis, } \\
\text { cough and asthma, toxic }\end{array}$ \\
\hline 64 & $\begin{array}{l}\text { Ketela } \\
\text { rambat }\end{array}$ & Ipomoea batatas Poir & Euphorbiaceae & Stem, leaf & Colds \\
\hline 65 & Jarak pagar & Jatropa curcas L. & Euphorbiaceae & Latex, fruit & Protection of teeth,toxic \\
\hline 66 & Jodium & Jatropha multifida $\mathrm{L}$. & Euphorbiaceae & $\begin{array}{l}\text { Latex (whole } \\
\text { plant) }\end{array}$ & Cure wounds, toxic \\
\hline 67 & Pohong & $\begin{array}{l}\text { Manihot esculenta } \\
\text { Crantz. }\end{array}$ & Euphorbiaceae & Root, leaf, tape & Vitality, alkoholic, tape \\
\hline 68 & Jarak kepyar & Ricinus communis $\mathrm{L}$. & Euphorbiaceae & $\begin{array}{l}\text { Latex, seed } \\
\text { (whole plant) }\end{array}$ & $\begin{array}{l}\text { Toxic, protection of teeth, herbs } \\
\text { (seasoning) }\end{array}$ \\
\hline 69 & Klanding & $\begin{array}{l}\text { Albitzia lophanta } \\
\text { (Wild.) Bth. }\end{array}$ & Fabaceae & Latex, fruit, seed & Appetite wound, vitality \\
\hline 70 & Dadap & $\begin{array}{l}\text { Erythrina variegata } \\
\text { L. }\end{array}$ & Fabaceae & Leaf & Flu remedy,colds \\
\hline 71 & Pronojiwo & $\begin{array}{l}\text { Euchresta horsfieldii } \\
\text { (Lesch.) Benn }\end{array}$ & Fabaceae & Stem, seed & $\begin{array}{l}\text { Vitality, gastric troubles, } \\
\text { impotence }\end{array}$ \\
\hline 72 & Toro & $\begin{array}{l}\text { Leucaena } \\
\text { leucacephala (Lamk.) } \\
\text { De Wit. }\end{array}$ & Fabaceae & $\begin{array}{l}\text { Young leaf, } \\
\text { latex, fruit, seed }\end{array}$ & $\begin{array}{l}\text { Itch,gastric pains, appetite, } \\
\text { protection of teeth }\end{array}$ \\
\hline 73 & Riwilkop & Mimusa pudica L. & Fabaceae & Leaf & $\begin{array}{l}\text { Gastric pains, protection of } \\
\text { teeth }\end{array}$ \\
\hline 74 & Benguk & $\begin{array}{l}\text { Mucuna pruriens (L.) } \\
\text { DC. }\end{array}$ & Fabaceae & Seed & Stimulans \\
\hline 75 & Pete & $\begin{array}{l}\text { Parkia speciosa } \\
\text { Hassk. }\end{array}$ & Fabaceae & Seed & Appetite, stimulants \\
\hline 76 & Buncis & Phaseolus vulgaris L. & Fabaceae & $\begin{array}{l}\text { Young leaf, fruit, } \\
\text { seed }\end{array}$ & Sprue \\
\hline 77 & Ercis & Pisum sativum L. & Fabaceae & Leaf, fruit, seed & Stimulans \\
\hline 78 & Asam jawa & Tamarindus indica $\mathrm{L}$. & Fabaceae & Fruit & $\begin{array}{l}\text { Cough, whitish, } \\
\text { purification,sprained }\end{array}$ \\
\hline 79 & Rukem & $\begin{array}{l}\text { Flacourtia rukam } \\
\text { Zoll. \& Mor }\end{array}$ & Flacourtiaceae & Leaf & Gonorrhea \\
\hline 80 & Grinting & $\begin{array}{l}\text { Cynodon dactylon } \\
\text { Pers. }\end{array}$ & Gramineae & Stem & Injury of bone \\
\hline 81 & Alang-alang & $\begin{array}{l}\text { Imperata cylindrica } \\
\text { (L.) Beauv. }\end{array}$ & Gramineae & Rhyzomes & $\begin{array}{l}\text { Wound,fever, to treat } \\
\text { dysfunctional organs of cattle }\end{array}$ \\
\hline 82 & Padi & Oryza sativa L. & Gramineae & Flower, seed & $\begin{array}{l}\text { Shampoo,facilitating } \\
\text { themother'smilk, sprain, bone } \\
\text { fractures }\end{array}$ \\
\hline 83 & Tebu & $\begin{array}{l}\text { Sacharum } \\
\text { officinarum L. }\end{array}$ & Gramineae & Stem & $\begin{array}{l}\text { Stimulans, diabetes mellitus, } \\
\text { fever, to treat dysfunctional } \\
\text { organs of cattle,improvement of } \\
\text { appetite and in the treatment of } \\
\text { abdominal pain }\end{array}$ \\
\hline 84 & Gandum & Zea mays L. & Gramineae & Young fruit & $\begin{array}{l}\text { Smallpox,urinary disorders, } \\
\text { bladder cleaning and kidney } \\
\text { disorders }\end{array}$ \\
\hline 85 & Sereh & $\begin{array}{l}\text { Adropogon nardus } \\
\text { DC. }\end{array}$ & Gramineae & Leaf, rhizomes & Cough, fever \\
\hline 86 & Danglu & $\begin{array}{l}\text { Engelhardia spicata } \\
\text { L. }\end{array}$ & Juglandaceae & Stem, flower & Protection of eye \\
\hline 87 & Permenan & Mentha arvensis $\mathrm{L}$. & Labiatae & Whole plant: root & Vitality \\
\hline 88 & Kemangi & Oscimum basilicum & Labiatae & Young stem, & Vitality, male fertility, \\
\hline
\end{tabular}




\begin{tabular}{|c|c|c|c|c|c|}
\hline 89 & Keningar & $\begin{array}{l}\mathrm{L} . \\
\text { Cinnamomum } \\
\text { burmanii } \mathrm{Bl} \text {. }\end{array}$ & Lauraceae & $\begin{array}{l}\text { leaf, flower } \\
\text { stem }\end{array}$ & $\begin{array}{l}\text { perfumedbody } \\
\text { Vitalitas, cold, nose infections, } \\
\text { common cold }\end{array}$ \\
\hline 90 & Sintok & $\begin{array}{l}\text { Cinnamomum sintoc } \\
\text { Bl. }\end{array}$ & Lauraceae & Stem & Tuberculosis, vitality \\
\hline 91 & Apokat & $\begin{array}{l}\text { Persea americana } \\
\text { Miller. }\end{array}$ & Lauraceae & Fruit & $\begin{array}{l}\text { Sprue, smooth bowel } \\
\text { movement, hemorrhoid }\end{array}$ \\
\hline 92 & $\begin{array}{l}\text { Bawang prei, } \\
\text { tropong }\end{array}$ & Alium fistulosum L. & Liliaceae & Stem, leaf & $\begin{array}{l}\text { Tuberculosis.,tonic, } \\
\text { aphrodisiac, diuretic, } \\
\text { carminative, appetizer, } \\
\text { antispasmodic, diarrhea, } \\
\text { dysentery and rheumatism. }\end{array}$ \\
\hline 93 & $\begin{array}{l}\text { Bawang } \\
\text { putih }\end{array}$ & Alium sativum $\mathrm{L}$. & Liliaceae & Stem, leaf & $\begin{array}{l}\text { Hypertension, cold, } \\
\text { cough,tuberculosis, diureticum, } \\
\text { panu/tinea versicolor, headache }\end{array}$ \\
\hline 94 & Lidah buaya & Aloe vera Mill. & Liliaceae & Leaf & $\begin{array}{l}\text { Shampoo, body weakness and } \\
\text { in the treatment of pimples or } \\
\text { acne. }\end{array}$ \\
\hline 95 & Pandan suji & $\begin{array}{l}\text { Pleumele angustifolia } \\
\text { (Roxb.) N.T.Brown }\end{array}$ & Liliaceae & Leaf & Food color \\
\hline 96 & Lidah mertua & $\begin{array}{l}\text { Sansevieria } \\
\text { trifasciata Prain }\end{array}$ & Liliaceae & Leaf & Smokeabsorber \\
\hline 97 & Mladean & Loranthussp. & Loranthaceae & Whole plant & Cancer \\
\hline 98 & Jamur impes & $\begin{array}{l}\text { Calvatia bovista }(\mathrm{L} .) \\
\text { Van Overeem }\end{array}$ & Lycoperdaceae & Fruit & Itch/toxic, cancer \\
\hline 99 & Pacar & Lawsonia inermis L. & Lythraceae & leaf & Itch, nail polish \\
\hline 100 & Locari & $\begin{array}{l}\text { Michelia champaca } \\
\text { L. }\end{array}$ & Magnoliaceae & Fruit & Perfumedbody, ritual \\
\hline 101 & Waron & $\begin{array}{l}\text { Abelmonchus } \\
\text { moschatus Medik. }\end{array}$ & Malvaceae & Flower & Bitten by insect, skin allergies \\
\hline 102 & Kerut, garut & $\begin{array}{l}\text { Marantha } \\
\text { arundinacea } \mathrm{L} \text {. }\end{array}$ & Marantaceae & Rhyzomes & Powder skin, vitality \\
\hline 103 & Senggani & $\begin{array}{l}\text { Melastoma } \\
\text { polyanthum Bl. }\end{array}$ & Melastomaceae & Leaf & Highblood pressure drugs \\
\hline 104 & Mindi & Melia azedarach L. & Meliaceae & Leaf, fruit & $\begin{array}{l}\text { Toxic, skin infection, skin } \\
\text { diseases. }\end{array}$ \\
\hline 105 & Mahoni & $\begin{array}{l}\text { Switenia mahagoni } \\
\text { Jacq. }\end{array}$ & Meliaceae & Fruit, seed & Headache, encok \\
\hline 106 & Nangka & $\begin{array}{l}\text { Artocarpus } \\
\text { heterophyllus Lamk. }\end{array}$ & Moraceae & Young fruit & Dysentery \\
\hline 107 & Ringin & Ficus benyamina $\mathrm{L}$. & Moraceae & Leaf, fruit & Blood purifications, ritual \\
\hline 108 & Lo & $\begin{array}{l}\text { Ficus glomerata } \\
\text { Roxb. }\end{array}$ & Moraceae & Fruit & Dysentery,gonorrhea \\
\hline 109 & Awar-awar & Ficus septica Burm.f. & Moraceae & Leaf, fruit & $\begin{array}{l}\text { Asthma,urinary problems, } \\
\text { constipation and vomiting }\end{array}$ \\
\hline 110 & Besaran & Morus alba $\mathrm{L}$. & Moraceae & Fruit & $\begin{array}{l}\text { Vitality, bad thorax, stomach } \\
\text { worms, demam, hemorrhoid }\end{array}$ \\
\hline 111 & Kelor & $\begin{array}{l}\text { Moringa oleifera } \\
\text { Lamk. }\end{array}$ & Moringiaceae & Leaf & Tuberculosis, headache \\
\hline 112 & Pisang & Musa paradisiaca $\mathrm{L}$. & Musaceae & $\begin{array}{l}\text { Fruit, latex, } \\
\text { flower }\end{array}$ & $\begin{array}{l}\text { Itch, dysentery, hemorrhoid, } \\
\text { ritual }\end{array}$ \\
\hline 113 & Kayu putih & $\begin{array}{l}\text { Eucalyptus alba } \\
\text { Reinw. ex Bl. }\end{array}$ & Myrtaceae & Leaves, seeds & $\begin{array}{l}\text { Cold, cough, throat lozenges, } \\
\text { malaria and toothache }\end{array}$ \\
\hline 114 & Jambu air & $\begin{array}{l}\text { Eugenia aquea } \\
\text { Burm.f. }\end{array}$ & Myrtaceae & Fruit & Vitality \\
\hline 115 & Cengkeh & $\begin{array}{l}\text { Eugenia aromatica } \\
\text { O.K. }\end{array}$ & Myrtaceae & Flower & Protection of teeth, cygarette \\
\hline 116 & Salam & Syzygium polyanthum & Myrtaceae & Leaf, fruit & $\begin{array}{l}\text { Rheumatism, body weakness } \\
\text { and in the treatment of pimples }\end{array}$ \\
\hline 117 & Poo & $\begin{array}{l}\text { Melaleuca } \\
\text { leucadendron L. }\end{array}$ & Myrtaceae & Stem, leaf & $\begin{array}{l}\text { Common cold, nose infections, } \\
\text { tuberculosis }\end{array}$ \\
\hline 118 & Jambu wer & Prunus persica Zieb. & Myrtaceae & Young leaf, & Dysentery, improvement of \\
\hline
\end{tabular}


\& Zucc.

119 Jambu klutuk Psidium quajava L.

Myrtaceae

young fruit

Leaf, fruit

\begin{tabular}{|c|c|c|}
\hline 120 & Blimbing & Averhoa carambola $\mathrm{L}$. \\
\hline 121 & Tapak kuda & Oxalis corniculata $\mathrm{L}$. \\
\hline 122 & $\begin{array}{l}\text { Pandan } \\
\text { wangi }\end{array}$ & $\begin{array}{l}\text { Pandanus } \\
\text { amaryllifolius Roxb. }\end{array}$ \\
\hline & Meniran & Phyllanthus ninuri L. \\
\hline & Katu & $\begin{array}{l}\text { Sauropus androgynus } \\
\text { (L.) Merr. }\end{array}$ \\
\hline & Pinus & $\begin{array}{l}\text { Pinus merkusii Jung \& } \\
\text { de Vries }\end{array}$ \\
\hline & Sirih & Piper betle L. \\
\hline
\end{tabular}

$\begin{array}{ll}\begin{array}{ll}\text { Oxalidaceae } \\ \text { Oxalidaceae } \\ \text { Pandanaceae }\end{array} & \begin{array}{l}\text { Fruit } \\ \text { Leaf }\end{array} \\ \begin{array}{ll}\text { Phyllanthaceae } \\ \text { Phyllanthaceae }\end{array} & \begin{array}{l}\text { Leaf, fruit } \\ \text { Whole plant }\end{array} \\ \text { Pinaceae } & \text { Bark, resin } \\ \text { Piperaceae } & \text { Whole plant }\end{array}$

Akar wangi

Polygala paniculata

L.

128 Paku jangan

Diplazium esculentum

Swartz.

129

Paku sarang

Drynaria quercifolia

J.Sm.

130 Jamur kayu

Ganoderma cochlear

(B1. et Nees Murrill.

131 Delima

132 Stroberi

133 Melati

134 Apel

135 Mawar

136 Grunggung

137 Kina

138 Kopi

139 Mengkudu

140 Simbukan

141 Jeruk nipis

Jeruk bali

Jeruk keprok

Punica granatum $\mathrm{L}$.

Fragaria fista $\mathrm{L}$.

Jasmicum sambac Ait.

Pyrus malus L.

Rosa multiflora

Thunb.

Rubus rosaefolius J.E.

Smith

\section{Cinchona ledgeriana}

Moens.

Coffea arabica L.

Morinda citrifolia L.

Paederia scandens

(Lour.) Merr.

Citrus aurantifolia

(Ch.\&P.) Sw.

Citrus maxima

(Burm.) Merr.

Jeruk manis Citrus sinensis Osb.

Lengkeng

Lerak

Sawo

Jamur grigit

\section{Lechi sinensis Sonn}

Sapindus rarak D.C.

Achras zapota L.

Schizophyllum alneum

(L.) Schr;

Schzophyllum

commune

149 Rumput kuda Selaginella ornata

Spring

150 Cubung

tingkat

Brugmansia candida

Pers.

$151 \quad$ Cubung
Polygalaceae Root, leaf

Polypodiaceae

Polypodiaceae

Polyporaceae

Punicaceae

Rosaceae

Rosaceae

Rosaceae

Rosaceae

Rosaceae

Rubiaceae

Rubiaceae

Rubiaceae

Rubiaceae

Rutaceae

Rutaceae

Rutaceae

Fruit

Rutaceae

Fruit

Sapindaceae Fruit

Sapindaceae Fruit

Sapotaceae Young fruit

Schizophyllaceae Fruit body

Selaginellaceae

Whole plant

Solanaceae

Leaf, fruit, flower

Solanaceae

\section{Brugmansia suaveolens Barcht. \&}

Leaf, fruit, flower appetite, and stomach problems

Dysentery, dengue fever, improvement of appetite, and stomach problems, dengue fever Hypertension

Influenza

Foot aromatic flavoring, body, ritual

Cough

Facilitatebreastfeeding

Burns and scalds, boils, cough and gastric troubles

Protection of teeth, nosebleed, blood purification, used for bath after delivery for body care, skin allergies

Bronchitis, itch, cought

Dysentery

Dysentery

Skin diseases, used for bath after delivery for body care Vitality, wormy, dysentery

Sprue disentery

Deodorizer body

Vitality, sprue

Deodorizer body, ritual

Sprue, astringens

Malaria fever

Hipertention, used in stomach ache, headache

Diabetes mellitus, hipertention Bloated stomach, headache

In the treatment of asthma, cough, tuberculosis

Vitality,toothpowder for teeth diseases and in infections. Sprue, toothpowder for teeth diseases and in infections. Sprue, toothpowder for teeth diseases and in infections. Vitality Soap, shampoo, iradicate insect Dysentery Vitality

\section{Dysentery}

Gonorrhea,used to reduce general body inflammation, intoxication, loss of appetite Gonorrhea,eye medicationused to reduce general body 
Presl.

$\begin{array}{lll}152 & \begin{array}{l}\text { Lombok } \\ \text { kriting }\end{array} & \text { Capsicum anuum } \mathrm{L} . \\ 153 & \begin{array}{l}\text { Pedesan, } \\ \text { rawit }\end{array} & \text { Capsicum frutescent } \\ \text { L. } \\ \text { Kangkung }\end{array} \quad \begin{aligned} & \text { Ipomoea aquatica } \\ & \text { Fosrk. }\end{aligned}$

161 Kentang

162 Teh

163 Adas

164 Purwoceng

176 Kunyit

177 Temu lawak

178 Kunci

179 Kencur

Curcuma xanthorhiza
Lycopersicum esculentum Mill.

Nicotiana tabacum L.

Physalis heterophyla L., Physalis minima L.

Solanum capiscatrum L. Solanum nigrum L.

Solanum torvum $\mathrm{Sw}$

Solanum tuberosum L. Solanaceae

Camelia sinensis (L.)

O.K.

Foeniculum vulgare

Mill.

Pimpinella pruacan

Molkenb.

pepagan

Centella asiatica Urb.

Usnea dasypoga

(Acharius) Nylander

Vaccinum

varingiaefolium (B1.)

Miq

telekan

Lantana camara $\mathrm{L}$.

Stachytarpetha indica

(L.) Vahl

Vitis vinifera $\mathrm{L}$.

Volvaria volvacea

(Bull.) Fries

Alpinia galanga $(\mathrm{L}$. Wild.

\section{Amomum}

cardanomum $\mathrm{L}$.

Canna edulis Ker.

Curcuma aeruginosa

Roxb.

Curcuma domestica

Val.

L.

\section{Kaempferia} angustifolia Rosc.

Kaempferia galanga
Solanaceae

Theaceae

Umbelliferae

Umbelliferae

Umbelliferae

Usneaceae

Fruit

Fruit and leaf

Young stem, leaf

Fruit

Young

stem,leaf

Whole plant

Fruit

Leaf, fruit

Fruit

Stem

Leaf

Whole plant

Whole plant

Leaf

Fruit

Vaccinaceae

Verbenaceae

Verbenaceae

Vitaceae

Volvariaceae

Zingiberaceae

Fruit

Whole plant

Whole plant

Fruit

Fruit

Rhyzomes

Zingiberaceae

Rhyzoma,

fruit, seed

Zingiberaceae

Rhizomes, leaf

Rhyzomes

Rhyzomes

Zingiberaceae

Rhyzomes

Zingiberaceae

Zingiberaceae

Rhyzomes

Rhyzomes inflammation, intoxication, loss of appetite

Cold, cough, vitality, stimulans, sweatbullets

Flu, vitality, stimulans, sweatbullets

Smooth bowel movements, hemorrhoid,constipation, control dandruff,skin diseases, constipaton, vomitting Eyepain medications, Sprue, ambeien (hemorrhoid)

Bitten by asnake,tooth ache,malaria(mosquito) Wound, medicine, sprue, diabetes mellitus Sprue

Stimulants, fasility urinare, used for abortionand painful secretions from ears

Stimulans, appetiteabnormal and painful secretions from ears Vitality, appetite, ritual Vitality, urinary,skin cleansers, dysentery

Stimulans, fever, cought, in the treatment of asthma.

Vitality for men, diabetes mellitus

Astma,bladder stones

Vitality,Javanes

traditionalherbal people Vitality

Toxic, leaf paste applied to treat allergy, athlete's foot and ringworm.

Vitality, facilitatingurination Skin diseases, vitality

Liver disease, fungi. rheumatism, body weakness and in the treatment of pimples or acne.

Analgetic, tuberculosis

Powder skin, dysentery Javanes Traditional herbal, helminthic

Fungi, liver, food color

Hipertention, liver, cancer

Vitality

In the treatment of asthma, 


\begin{tabular}{ccllll}
180 & Lempuyang & $\begin{array}{l}\text { L. } \\
\text { Zingiber aromaticum }\end{array}$ & Zingiberaceae & Rhyzomes & $\begin{array}{l}\text { broken bones, sprains } \\
\text { Vitality (Javanese traditional } \\
\text { herbal) }\end{array}$ \\
181 & Jae, jae wono & $\begin{array}{l}\text { Zingiber } \text { officinale } \\
\text { Roxb. }\end{array}$ & Zingiberaceae & Rhyzomes & $\begin{array}{l}\text { Vitality, blood purification, } \\
\text { sprained }\end{array}$ \\
\hline
\end{tabular}

The results showed that part of the plant used as an ingredients medicine consistingof rhizomes, sap, roots, stem bark, leaf, flower, fruit and seed. Total of 181 plant species determinate of Tengger and Java people existing in the region. The indigenous knowledge and useful medicine of plants is magnificent and one species dancukan (Gardenia palmata) very toxic. Family ethnomedicine plants that have large members includes Euphorbiaceae (8 species), Fabaceae (10 species), Zingiberaceae (10 species), Solanaceae (12 species) and Asteraceae (15 species).On the other hand family importance e.g. Rosaceae (Pyrus malus), Araceae (Acorus calamus), Myrtaceae (Prunus persica), Musaceae (Musa paradisiaca), Caricaceae (Carica papaya, Carica pubescent), Apiaceae (Foeniculum vulgare). The number of plants used to treat more than 60 diseases. The necessity of traditional Tengger people'sknowledge driven by the needs formal life in their environment. Related to the needs they have to manage, used, controled and market.

The research of local knowledge should be followed with the knowledge of ethics, which in turn flows into the validity and regulatory framework. Local knowledge is the basic which is very useful to support scientific research (ethics) and as the key in the developingpolicies in plants with pharmacological value, ultimately for the national and international markets. The traditional knowledge of Tengger tribe about the medicine becomethe important and very valuable in the development of the pharmacology fields.

The less of government attention, foolishness, poverty, ignorance and lack of modern health facilities, so the most local people use traditional medicine in everyday life. Most of special Tengger people gain strength with mantra (suwuk), both in the compounding and the implementation of their traditional medicine. A knowledge of how public compounding medicinal plant materials are to combat against various diseases, although regional differences within a tribe into a traditional knowledge that very important.Recentlythe use of medicinal plants by the Tengger tribe and Java people beginning to be, due to less practical, especially on the younger generation.Based on the results of this study conducted on Ethno-medicine local people as follows: (a) Traditional knowledge of medicinal plants. (b) Compounding techniques of medicine, selection types of plant, organs and types of diseases.

\section{Conclusions}

An ethnomedecinal survey was carrying in district Poncokusumo Malang, Province East Java, Indonesia for documentation of importan plants diversity and information from local people. Total of 181 plant species determinate of Tengger and Java people existing in the region. The indigenous knowledge and useful medecine of plants is magnificent. Family ethnomedicine plants that have large members includes Euphorbiaceae ( 8 species), Fabaceae (10 species), Zingiberaceae (10 species), Solanaceae (12 species) and Asteraceae (14 species).The number of plants used to treat more than 60 diseases. The treatment done by a medicine man or shaman from Tengger people by ritual treatment with called "Suwuk".Qualitative approach (emic) must be followed research ethic with intensive, and phytochemicals present identity. This study can be references to Perhutani, Bromo Tengger Semeru National Park (BTS NP), local people, companies, and pharmacology for further development.

\section{Acknowledgements}

First of all the researcher gives special thanks to the Herbarium Departementof Biologi(H Bio Unibraw) for their help and co-operation extended in several ways. The researcheralso gives special acknowledgement to Competitive Eminent Research Projects of Higher Education PHK Brawijaya University, so that the research could be conducted. The researcher also give thanks to the local communities of local people ddistrict Poncokusumo, Malang, East Java, Indonesia. Last but not least, we also thank for the supportfrom the leader BTS NP, Perhutani and of the leader local and Tengger people in Malang. Recommendations Qualitative approach (emic) must be followed research ethic with intensive, and phytochemicals present identity. This study can be references to Perhutani, Bromo Tengger Semeru National Park (BTS NP), local people, companies, and pharmacology for further development.

\section{Conflict of interest}

None to declare. 


\section{References}

Backer CA and RC Bakhuizen van den Brink, f, 1968. Flora of Java.Vol. I, II, III (Spermatophytes Only) NVP. Noordhoff. Groningen-The Netherlands.

Batoro J, 2015. Pengelolaan Lingkungan Dengan Pendekatan Etnobiologi-Etnobotani. Universitas Brawijaya. Penerbit UB Press. pp. 1-106.

Batoro J, 2015. Mengenal Jenis-Jenis Tumbuhan dan Kegiatan Masyarakat Desa Hutan di Kawasan Bromo Tengger Semeru. Penerbit Selaras Malang.

Chinery HM, 1982. Encyclopedie Van De Natuur Flora en Fauna VanNetherland en Europa Vrooom Dreesmann.

Cotton CM, 1996. Ethnobotany: Principle and Applications. John Wiley \&Sons. Chichester, New York, Brisbane, Toronto, and Singapore.

De Vogel EF, 1987. Manual of Herbarium Taxonomy (Theory and Practice). UNESCO. Jakarta.

Hoffman B and T Gallaher, 2007. Importance Indices in Ethnobotany. Ethnobotany Research \& Applications 5:201-208.

Lemmens RHMJ,1989. Plant Resource of South East Asia, Wageningen The Netherlands.

Rambo AT,1983. Conceptual Approaches to Human Ecology. East-West Environment and Policy Institute, East-West Center, Honolulu, Hawaii. USA. Research Report 14: 6. pp. 1-26.

Rifai MA,1994. A discourse on Biodiversity Utilization in Indonesia. Herbarium Bogoriense, Puslitbang Biologi-LIPI, Bogor, Indonesia.

Sheil D, RK Puri, I.Basuki, Mvan Heiizt, MWan, N Liswanti, Rukmiyati, MA. Sardjono, L. Samsoedin, K Sudiyasa, Chrisandini, E Permana, EM Angi, F Gatzweiler, B Johnson and Wijaya, 2004. Exploring biological diversity, environment and local people's perspectives in forest landscapes. Methods for a multidisciplinary landscape assesment. CIFOR, Bogor. Indonesia.

Stibbe DG and UM Uhlenbeck 1921. Tengger, Encyclopedie van Nederlandch-IndieLeiden.

Tyler VE, LR Brady and JE Robbers. 1976. Pharmacognosy, Printed in the United State of, America.

Waluyo EB, 2004. Pengumpulan Data Etnobotani. Training Database and Networking Biodiversity-NIBN. Puslit Biologi-LIPI. pp. 77-104.

Westphal E and PCM Jansen, 1989. Plant Resource of South East Asia Pudoc Wageningen. 